\title{
Analytical Study of Resistive Transition Curves in a $\mathrm{SrAuSi}_{3}$ Superconductor
}

\author{
Tsugio Hamada \\ Department of Electrical and Information Engineering, N.I.T., Miyakonojo College \\ Miyazaki 885-8567, Japan \\ Yuki Sakamoto \\ Department of Electrical and Information Engineering, N.I.T., Miyakonojo College \\ Miyazaki 885-8567, Japan \\ Masatake Masudome \\ Department of Electrical and Information Engineering, N.I.T., Miyakonojo College \\ Miyazaki 885-8567, Japan
}

\begin{abstract}
The resistive transition curves in a $\mathrm{SrAuSi}_{3}$ superconductor are analytically calculated by flux creep theory. The resistive transition carves in magnetic fields have been well implemented to be similar to the experimental ones. The $\mathrm{SrAuSi}_{3}$ superconductor is so inferior to those of the high- $\mathrm{T}_{\mathrm{c}}$ superconductors both in the critical current density, $J_{c}$, and the upper critical field, $B_{\mathrm{c} 2}$, from the analytical results. The both $J_{\mathrm{c}}$ and $B_{\mathrm{c} 2}$ values in $\mathrm{SrAuSi}_{3}$ superconductor should be improved for the power applications.
\end{abstract}

Keywords - SrAuSi $i_{3}$ superconductor, flux creep theory, resistive transition curves, application level .

\section{INTRODUCTION}

A new type superconductor had been discovered by M.Isobe et al in 2014 [1]. The superconductor has a strange characteristic containing gold atom in its structure. This is because the gold atom is chemically most stable atom in comparison with other atoms. The interest of this superconductor by the many scientists is therefore mainly the superconducting mechanism. However, an application for the power equipment is required the high critical current density at high magnetic fields and/or at high temperatures. The other parameters such as the upper critical field, $B_{c 2}$, and the critical current density, $J_{c}$, at $0 \mathrm{~K}$ are also estimated from the analyzed resistive transition characteristic using flux-creep theory in this paper.

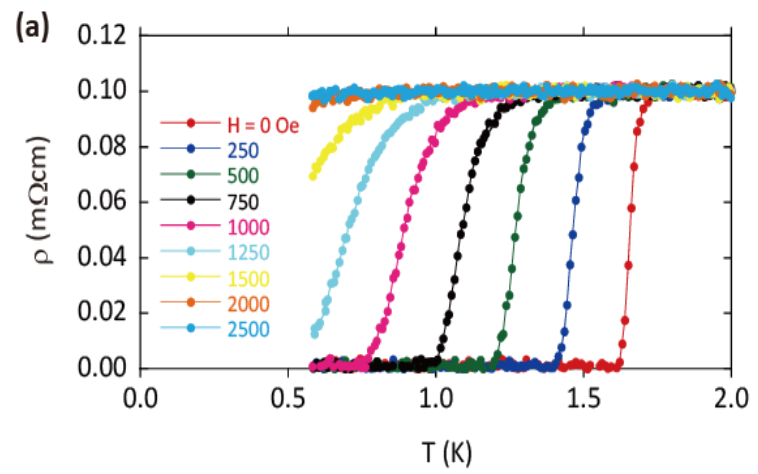

Figure1. Resistive and temperature curves of a $\mathrm{SrAuSi}_{3}$ superconductor under various magnetic fields [1]

Resistive transition caves in high- $T_{c}$ superconductors from the behavior of fluxoids had been reported by T.Matsushita [2]. Resistive transition characteristic of the superconductor for the magnetic field has moved to the lower temperature side by increasing the magnetic fields [1]. The authors have ever investigated superconducting properties such as the irreversibility field characteristic for many superconductors [3]. This resistive transition curves in the reference [1] is so similar to the metallic superconductor like a metal NbTi superconductor. The mechanism of resistive transition in the case of high- $T_{c}$ superconductors is not easy in comparison with that of the metal superconductors. 
The validity of the flux-creep theory for the new type superconductor is validated in the resistive tradition curves in magnetic fields. If the resistive transition characteristic in this superconducting system can be explained by the flux creep theory, the application for power equipment will be roughly predicted. First of all, the resistance temperature characteristic will be analyzed by using flux-creep theory in this paper. The obtained results are compared with the experimental one.

\section{FLUX CREAP-FLOW MODEL}

We assume a sinusoidal washboard potential for describing approximately the variation in the energy of flux bundle versus its position. The pinning potential, $V_{b}$, is proportional to the flux bundle, given as

$$
v_{b}=\left(g a_{f}\right)^{2} i_{44}
$$

for a bulk superconductor, where $g^{2}$ is the number of fluxoids inside the flux bundle, $\alpha_{f}$ is the fluxoids spacing, and $l_{44}$ is the longitudinal correlation length of the fluxoids. If the size of superconductor, $D$, is smaller than $l_{44}, \mathbb{V}_{6}$ is limited by $D$ and given by

$$
F_{0}=\left(g x_{f}\right)^{2} D
$$

According to the flux creep theory in which the size of superconductor is taken into account, the pinning potentials are given by

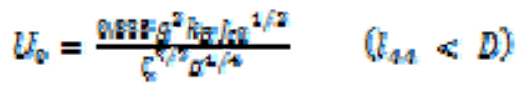

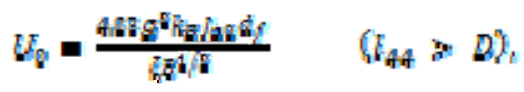

This leads to an expression (3) of the activation energy, $U$, as a function of the pinning potential, $U_{0}$, and the normalized current density, $j=J / J_{0}$, with $J_{c 0}$ denoting the virtual critical current density in the creep-free case. According to the flux creep theory $U_{0}$ is expressed in terms of $J_{c 0}$ [4]. The contribution from the flux creep to the induced electric field in given by

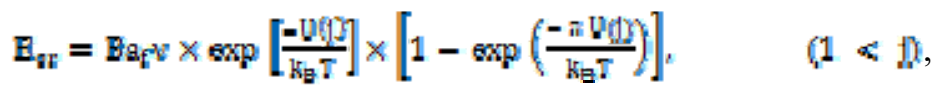

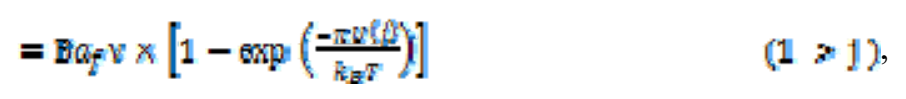

where $\mathrm{a}_{\mathrm{f}}$ is the fluxoid spacing and $v_{0}=\rho_{f} J_{c 0} / a_{f} B$ is the oscillation frequency of flux bundle and $\rho_{f}$ is the flux flow resistivity, for which the Bardeen-Steohen model is assumed: $\rho_{f}=\left(B / B_{c 2}\right) \rho_{n}$. Here, the temperature region for the measurement of resistance is very low temperature which is lower than liquid helium temperature. Therefore, the contribution from the flux creep is very small [5]. On the other hand, the contribution from the flux flow is

$$
\begin{aligned}
& E_{f f}=0, \quad \text { (1) } ; 0
\end{aligned}
$$

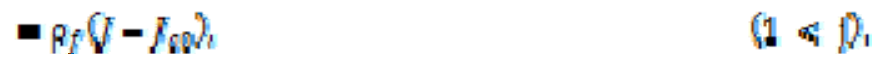

Here we approximate that the total electric field is simply given by $\mathrm{E}=E_{c r}+E_{f f}$.

It is seen from Fig. 1 that the critical temperature $T_{c}$ is locally fluctuated in in the specimen. Hence, we assume the distribution function of $\mathrm{T}_{\mathrm{c}}$ of the form:

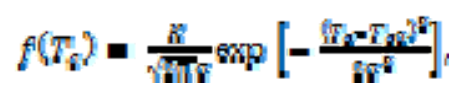

where $T_{c 0}$ and $\sigma$ are approximately the mean value and the standard deviation, respectively and $K=1$. Because of the distribution of $T_{c}$, the upper critical field and the flux pinning strength may be also distributed. However, the 
dependence of the upper critical field and the pinning strength on Tc is not considered.in this flux creep-flow model. We assume simply that $J_{c 0}$ varies as [4]

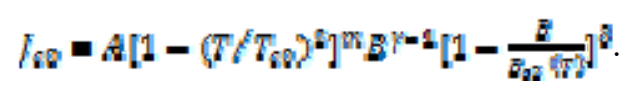

In the above $A$ is a constant. $\mathrm{T}_{\mathrm{c} 0}$ is the middle critical temperature, i.e., average temperatures of $\mathrm{T}_{\mathrm{c}}$ onset and $\mathrm{T}_{\mathrm{c}}$ offset. The values of $\mathrm{m}$ and $\gamma$ in Eq. (4) are obtained so that a good fit is obtained between this expression and the experimental results. $\delta=20$ is assumed in this calculation because of weekly pinning in the specimen. The variance of the critical temperature used in the calculation is determined from following. The Gaussian distribution is considered as the resistive transition wide shown in Fig. 2.

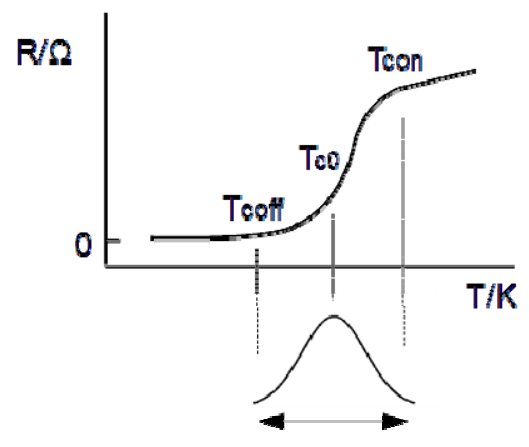

Figure 2. Schematic illustration for the decision of $\sigma$ from the resistive transition curve at zero field

As a result, the electric field is calculated by

$$
E=\int_{\sigma}^{*}\left(E_{s c}+E_{f f}\right) f\left(T_{\varepsilon}\right) \mathrm{d} \tau_{\varepsilon}
$$

The parameters used in this calculation are listed in Table 1 . The $J_{c}$ is determined using the electric field criterion of $1.0 \times 10^{-4} \mathrm{~V} / \mathrm{m}$. The variance of the critical temperature is determined from the wide of resistive transition curve at zero magnetic field.

\section{III.RESULTS AND DISCUSSION}

Table 1 Parameters used in the numerical calculation

\begin{tabular}{cccccccc}
$T_{\mathrm{c} 0}[\mathrm{~K}]$ & $B_{\mathrm{c} 2(0)}[\mathrm{T}]$ & $\rho_{\mathrm{n}}[\Omega \mathrm{m}]$ & $A(0)$ & $m$ & $\gamma$ & $\delta$ & $\sigma^{2}$ \\
\hline 1.67 & 0.25 & 0.01 & $5 \times 10^{4}$ & 10 & 0.3 & 2.0 & 0.004
\end{tabular}

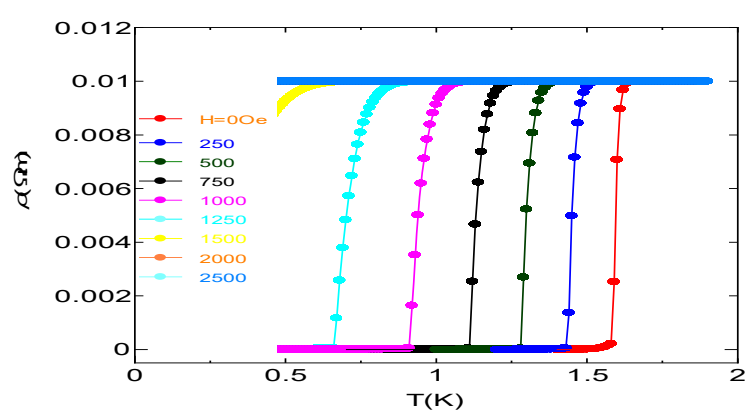

Figure 3. The calculated resistance-temperature carves used the flux-creep theory 
The theoretically calculated resistance transition carves are shown in Fig.3. The results calculated by the flux creep theory are well similar to the experimental ones.

The critical current density, $J_{c}$, and the upper critical field, $B_{c 2}$, are the key parameters for applications of all superconducting materials. High- $T_{c}$ superconductors which had been discovered 20 years before are better than that in metal superconductors about the upper critical fields. The upper critical fields in high- $T_{c}$ superconductors are easily over $50 \mathrm{~T}$ at $0 \mathrm{~K}$. However, the critical current density in high- $T_{c}$ superconductors is not enough to use for application in comparison with $\mathrm{NbTi}$ materials. Let us consider the critical current density and the upper critical field in the $\mathrm{SrAuSi}_{3}$ superconductor on the application for engineering from the results obtained. The $\mathrm{SrAuSi}_{3}$ superconductor, however, is so inferior to those of the high- $T_{c}$ superconductors both in the critical current density, $J_{\mathrm{c}}$, and the upper critical field, $B_{\mathrm{c} 2}$. The parameters used in this calculation are shown in Table 1 . The $B_{\mathrm{c} 2}(0)$ and $A(0)$ is also smaller than those in the ordinary superconductors. For example, schematic illustrations for $J-T$ plane and $B-T$ plane are shown in Fig.4. The solid lines in Fig.4 show schematically the $J_{\mathrm{c}}(T)$ or $B_{\mathrm{c} 2}(T)$ in each plane for ordinary superconductors. The dashed lines in each plane show the $J_{\mathrm{c}}(T)$ or $B_{\mathrm{c} 2}(T)$ in the $\mathrm{SrAuSi}_{3}$ superconductor, respectively. The values are too smaller than those in ordinary superconductors. By the way, the $B_{\mathrm{c} 2}(0)$ and $A(0)$ in the multifilamentary NbTi wire have roughly values of $15 \mathrm{~T}$ and $1.7 \times 10^{10} \mathrm{~A} / \mathrm{m}^{2}$, respectively[6].
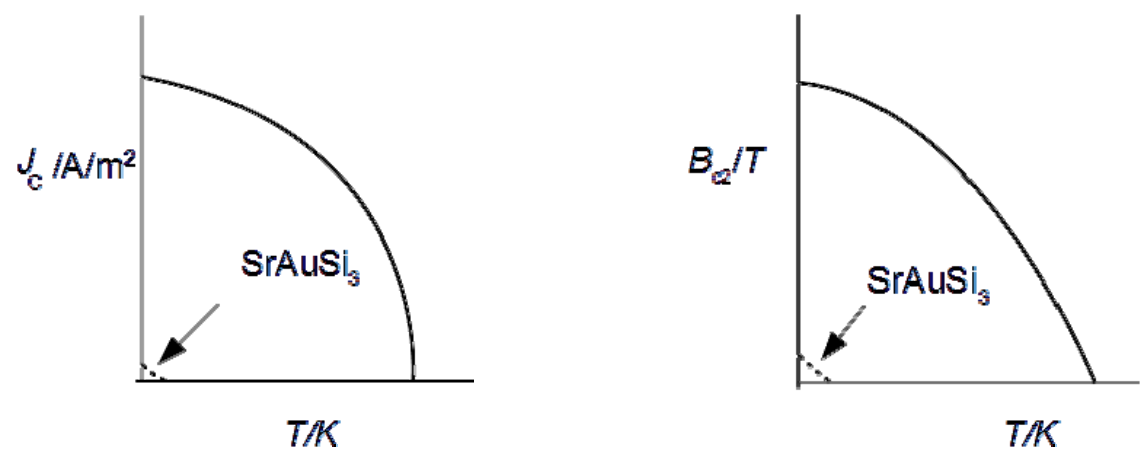

Figure 4. Schematic illustration of comparisons with $J_{\mathrm{c}}$ and $B_{\mathrm{c} 2}$ in a $\mathrm{SrAuSi}_{3}$ superconductor and in the ordinary superconductor's ones

About the critical current density, it is possibility to be affected by the irreversibility field or the irreversibility temperature. That is, reversible region is in the $B-T$ plane where is the superconducting state but $J_{\mathrm{c}}=0$. The fluxoid can be easily moved. That is, as the pinning is not effective in this region, effective pinning centers must make into the $\mathrm{SrAuSi}_{3}$ material of the superconductor. Therefore, much more detailed experimental investigation is necessary to clarify the responsible reason why the areas of superconducting state are narrower than those in ordinary superconductors. For the purpose, it is necessary to examine with the $\mathrm{SrAuSi}_{3}$ superconductor about many magnetic and/or temperature dependence data.

\section{IV.CONCLUSION}

The SrAuSi3 superconductor has been investigated in the resistance temperature transition curves in this paper using flux-creep theory. The results obtained are as follows:

(1) The analytically calculated resistance temperature transition carves are implemented by using the flux creep theory. The calculated transition curves are well similar to the experimental ones.

(2) $\mathrm{The}_{\mathrm{SrAuSi}}$ superconductor is so inferior to those of the high- $T_{c}$ superconductors both in the critical current density, $J_{\mathrm{c}}$, and the upper critical field, $B_{\mathrm{c} 2}$. The both values in the SrAuSi3 superconductor are not yet attained to the level of the applications.

\section{Acknowledgment}

The authors wish to express his thanks to the members of their group, especially Messrs. S.Tsuboyama, K.Simizu, S.Miyanaga, and S.Yoshimoto, who carried out much of the experimental and constructional works in spite of summer vacation, and in the middle of job hunting. 


\section{REFERENCES}

[1] M.Isobe, H.Yoshida, and K.Kimoto, Chemistry of Materials, vol.26 (2014), DOI: 10.1021/cm500032u

[2] T.Matsushita, "Flux Creep and Critical Currents in Oxide Superconductors", PHYSICA B vol.164, pp.150-159, 1990

[3] S.Hidaka, Y.Serita, and T.Hamada, "Resistive Transition of a weakly Pinned Nb-Ti Superconductor" Proc. $11^{\text {th }}$ International Symposium on Advanced Science and Technology in Experimental Mechanism, pp.126-129, 2016

[4] T.Matsushita, "On the flux bundle size in weakly pinned superconductors", Phsica C vol.217, pp.461-466, 1993.

[5] Y.Sakamoto, Graduation thesis of N.I.T. Miyakonojo College,pp.18-25, 2016

[6] K.Watanabe, H.Noto, and Y.Mutoh, "The upper critical fields in multifilamentaly NbTi alloy wires", TEION-KOUGAKU vol.26 No.2, pp.120-125, 1991 